University of Wollongong

Research Online

Faculty of Engineering and Information

Faculty of Engineering and Information

Sciences - Papers: Part A

Sciences

$1-1-2014$

\title{
Fault approximation tool for grid-connected inverter-interfaced distributed generators
}

Joel Kennedy

University of Wollongong, jtk847@uowmail.edu.au

Philip Ciufo

University of Wollongong, ciufo@uow.edu.au

Ashish Agalgaonkar

University of Wollongong, ashish@uow.edu.au

Follow this and additional works at: https://ro.uow.edu.au/eispapers

Part of the Engineering Commons, and the Science and Technology Studies Commons

Research Online is the open access institutional repository for the University of Wollongong. For further information contact the UOW Library: research-pubs@uow.edu.au 


\title{
Fault approximation tool for grid-connected inverter-interfaced distributed generators
}

\begin{abstract}
A novel fault approximation tool (FAT) is presented for use in distribution networks with a high penetration of inverter-interfaced distributed generation. The FAT does not rely on balanced operation nor an absolute reference point for voltage calculations, both of which are required for Newton-Raphson load flow calculations. Simulations show that the FAT provides an accurate representation of the transient and steady-state fault response for unbalanced faults when juxtaposed with a detailed small-signal model. The 'sub-transient' response of the inverter-interfaced distributed generation is omitted to remove data considered extraneous from the perspective of distribution line protection analysis. The FAT is not appropriate for analysing the efficacy of distributed generation local protection and is inappropriate for islanded applications. The FAT is designed explicitly for the purpose of utility line protection design and analysis.
\end{abstract}

\section{Keywords}

approximation, fault, tool, generators, grid, connected, inverter, interfaced, distributed

\section{Disciplines \\ Engineering | Science and Technology Studies}

\section{Publication Details}

J. Kennedy, P. Ciufo \& A. Agalgaonkar, "Fault approximation tool for grid-connected inverter-interfaced distributed generators," in Power Engineering Conference (AUPEC), 2014 Australasian Universities, 2014, pp. 1-6. 


\section{Fault Approximation Tool for Grid-Connected Inverter-Interfaced Distributed Generators}

\author{
Joel Kennedy \\ School of Electrical and \\ Computer Engineering \\ University of Wollongong \\ Wollongong, NSW, Australia \\ Email: jtk847@uowmail.edu.au
}

\author{
Phil Ciufo \\ School of Electrical and \\ Computer Engineering \\ University of Wollongong \\ Wollongong, NSW, Australia \\ Email: ciufo@uow.edu.au
}

\author{
Ashish Agalgaonkar \\ School of Electrical and \\ Computer Engineering \\ University of Wollongong \\ Wollongong, NSW, Australia \\ Email: ashish@uow.edu.au
}

\begin{abstract}
A novel fault approximation tool (FAT) is presented for use in distribution networks with a high penetration of inverter-interfaced distributed generation. The FAT does not rely on balanced operation nor an absolute reference point for voltage calculations, both of which are required for NewtonRaphson load flow calculations. Simulations show that the FAT provides an accurate representation of the transient and steadystate fault response for unbalanced faults when juxtaposed with a detailed small-signal model. The 'sub-transient' response of the inverter-interfaced distributed generation is omitted to remove data considered extraneous from the perspective of distribution line protection analysis. The FAT is not appropriate for analysing the efficacy of distributed generation local protection and is inappropriate for islanded applications. The FAT is designed explicitly for the purpose of utility line protection design and analysis.
\end{abstract}

\section{INTRODUCTION}

The increasing prevalence of inverter-interfaced distributed generation (IIDG) in distribution networks (DNs) has progressively undermined the efficacy of traditional line protection design philosophy. Many dynamic models have been developed in order to better understand the effects of IIDG on traditional protection design philosophy. However, the development of dynamic models is a time consuming process and IIDG unit design differs significantly across manufacturers. Utilities usually do not possess the time and resources to build a model for each different IIDG unit that is connected to a DN. To simplify to modelling process, the authors present a fault approximation tool (FAT) that utilities may adopt to ease the fault analysis of DNs with a high penetration of IIDG.

The development of dynamic models is a common topic within research. Authors [1], [2], [3], [4], [5] all present different control diagrams with the common goal of simulating a constant power grid-connected IIDG. An accurate representation of the control scheme of an IIDG

NOTICE: this is the authors' version of a work that was accepted for publication. Changes resulting from the publishing process, such as peer review, editing, corrections, structural formatting, and other quality control mechanisms may not be reflected in this document. Changes may have been made to this work since it was submitted for publication. A definitive version was subsequently published in the Proceedings of the Australasian Universities Power Engineering Conference (AUPEC), Sep. 2014 DOI:10.1109/AUPEC.2014.6966578 is of particular importance as there are no innate machine dynamics to govern the fault response. However, utilities may not have access to a detailed representation of an IIDG unit's control scheme and control data may be unavailable. Hence, from the utility perspective, the preferred modelling tool for a grid embedded with IIDG would require only limited information about the control scheme yet give a reasonable representation of the behaviour of the IIDG when exposed to a disturbance.

A simplified modelling tool is presented by [2] using Gauss-Seidel load flow. All phases are investigated independently, hence, it can be inferred that all neutral points are assumed to be earthed. There may also arise convergence issues for significant disturbances within the DN due to a poor selection of initial conditions. A similar tool is proposed by $\mathrm{Tu}$ in [6] using minor alterations to Newton-Raphson load flow, which will likely yield similar convergence issues to the tool proposed by [2]. The proposed FAT scheme contains allowances for floating neutral points by using Kirchoff's Voltage Law (KVL) in addition to Kirchoff's Current Law (KCL). Convergence issues are also much less likely using the proposed FAT as IIDG current outputs are used as the iterated variable; the initial values can be calculated using fault instant data without the need of an iterative process.

IIDG is generally not designed to sustain fault current continuously due to the low thermal inertia of the power electronic switches within the inverter [7]. The fault current capability of an inverter is dependent on the ability of the inverter to draw heat away from the power electronics during a sustained fault. Most contemporary IIDG units are not designed with the explicit intention of providing fault ride-through; hence, fault currents tend to be limited in magnitude and brief in duration. The authors of [8] find that fault current peak magnitudes can vary from 2 to 5 times rated current for 1 to 4.25 milliseconds. The magnitude of the fault current is determined by the grid conditions as well as the characteristics of the IIDG unit. The duration of the fault response is governed by the over-current protection of the IIDG unit. A single unified representation of the fault response for the short term transient of an IIDG unit is difficult as the thermal dynamics of the inverter must be accurately modelled in addition to the control and power dynamics of the IIDG 
and grid. The variety of fault current data gathered by [8] and the range of control schemes presented by authors [1], [2], [3], [4], [5] is testament to the impracticality of deriving a unified detailed model of short term transients. The FAT provides a means of approximating the fault response of IIDG for use in analysing DN line protection schemes without requiring an in-depth understanding of an IIDG unit's control structure.

The remainder of the paper is structured as follows. Section II details the FAT algorithm. Section III presents a comparison between the FAT and a detailed small-signal dynamic model. Section IV provides the conclusion with future recommendations.

\section{IIDG FAULT APPROXIMATION TOOL}

In order to establish a generalised model of IIDG units and verify the accuracy of the FAT, a dynamic model with the control scheme shown in Figure 1 was developed. A feedforward voltage is used to ensure fast voltage commutation of the IIDG unit. The control scheme contains a proportionalresonant (PR) controller which has two important advantages over commonly implemented proportional-integration controllers: PR controllers have zero steady state error and do not require knowledge of the AC side filter circuit parameters of the IIDH unit [9]. The control scheme also contains a phase locked loop (PLL) which determines the phase of the voltage at the IIDG unit's point of common coupling. Both the PR and PLL controllers have very fast tracking capabilities, thus are omitted in analysing the transient response of the FAT.

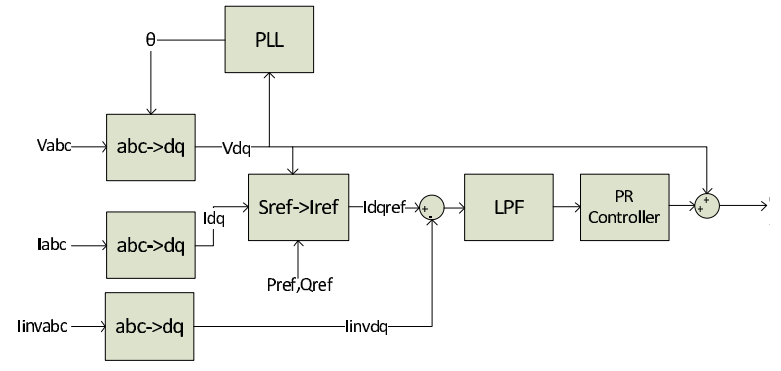

Fig. 1: IIDG Control Diagram

There are two features of the control scheme shown in Fig. 1 that are implemented when defining the dynamics of the FAT: a low pass filter (LPF) after the current controller and the feed-forward voltage signal. The LPF slows the response of the current reference which implies that the power exported by the IIDG at the instant of a fault will not necessarily be equal to the power set points during the transient response. The use of a feed-forward voltage signal implies that the voltage commutation of the IIDG is almost instantaneous. From the perspective of DN line protection device operation, the assumption of an instantaneous voltage shift is suitable as the anti-aliasing filters within protection devices attenuate high frequency transients [10].

The complete FAT algorithm is expressed as a logic diagram in Fig. 2. The algorithm begins by solving a

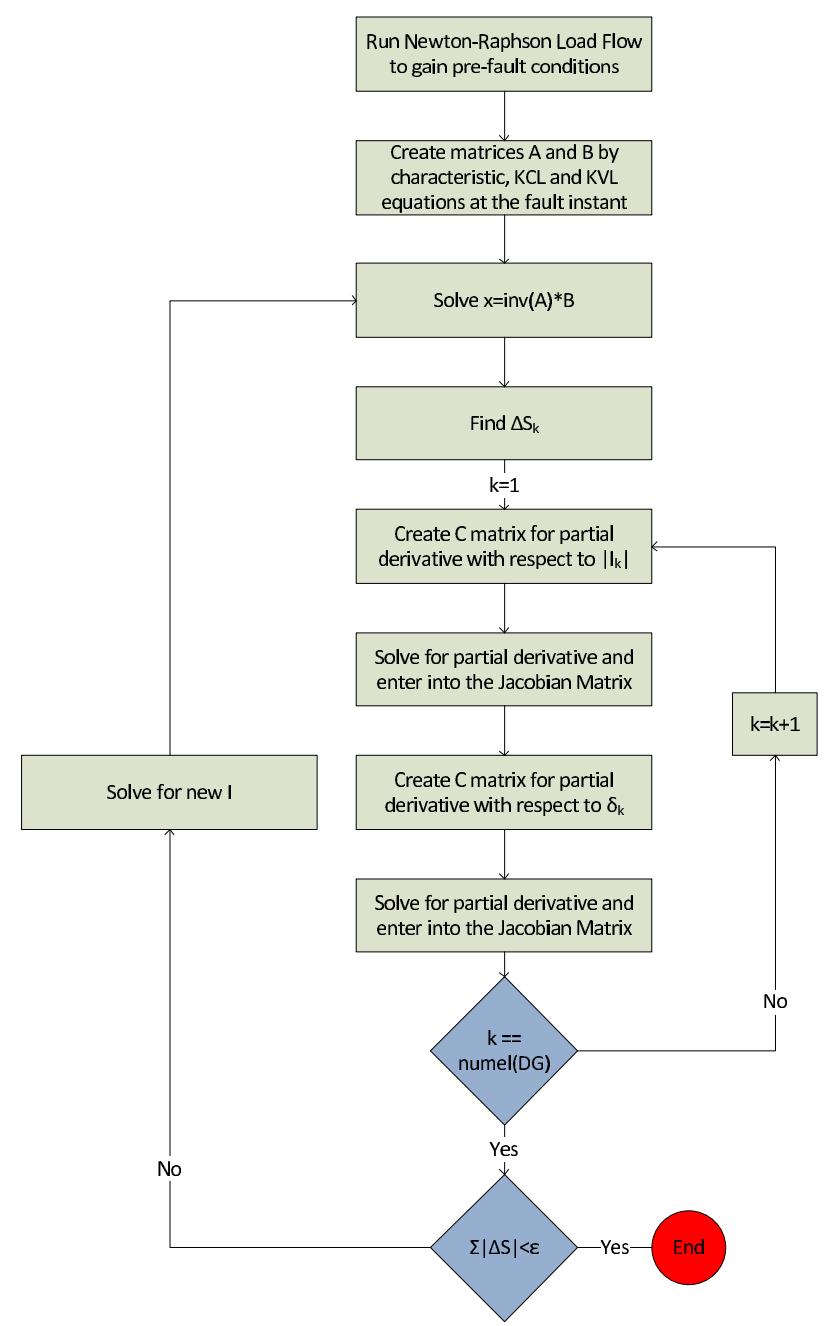

Fig. 2: FAT Flow Chart

Newton-Raphson load flow for the pre-fault condition. The Newton-Raphson algorithm can solve each phase independently but assumes that all neutral points are earthed. Newton-Raphson load flow is well established and will generally converge for a balanced DN under nominal conditions. Convergence issues are more likely to arise under fault conditions. Hence, the FAT implements a novel, iterative solver that converges upon IIDG currents instead of all non-slack node voltages as used in the Newton-Rapshon load flow solver.

The network model of $\mathrm{N}$ elements is then represented as a set of characteristic, KVL and KCL equations as shown in (1). An element is any phase of a source, line, load or transformer winding.

$$
A x=B
$$

Where: 


$$
\begin{gathered}
A=\left[\begin{array}{c}
\text { characteristic } \\
\text { KCL } \\
\text { KVL }
\end{array}\right] \\
x=\left[\begin{array}{lllllll}
V_{1} & I_{1} & V_{2} & I_{2} & \ldots & V_{N} & I_{N}
\end{array}\right]^{\prime} \\
B=[\text { constants }]
\end{gathered}
$$

All matrix elements contain vectors unless otherwise specified. The voltages in the $x$ matrix $V_{1}, V_{2}, \ldots$ are the potential differences across each element (there is no need for an absolute reference as used in the Newton-Raphson solver). The currents in the $x$ matrix $I_{1}, I_{2}, \ldots$ are the currents passing through each element. Each line of the characteristic, KCL and KVL matrices are of sizes $\mathbb{C}^{1 \times 2 N}$ and the constants matrix is of size $\mathbb{C}^{2 N \times 1}$. The characteristic and constant matrices of lines and loads are simple arrangements of Ohm's Law, (2);

$$
\left[\begin{array}{ll}
-1 & Z_{p}
\end{array}\right]\left[\begin{array}{l}
V_{p} \\
I_{p}
\end{array}\right]=[0]
$$

where $p$ is an line or load element. An infinite slack bus at element $p$ is represented as a constant voltage, (3).

$$
\left[\begin{array}{ll}
1 & 0
\end{array}\right]\left[\begin{array}{c}
V_{p} \\
I_{p}
\end{array}\right]=\left[V_{p}\right]
$$

Transformers can be connected in any configuration. However, the characteristic equation does not require a knowledge of external winding connections; rather, a three phase transformer is represented as three single-phase transformers. The determination of the winding connections and subsequent phase shifting is achieved through the KCL and KVL equations. Each transformer phase is treated as two separate elements $p$ and $q$ and hence requires two characteristic equations.

$$
\left[\begin{array}{cccc}
-1 / Z_{m} & 1+Z_{p} / Z_{m} & 0 & 1 / a \\
1 & -Z_{p} & a & a Z_{q}
\end{array}\right]\left[\begin{array}{c}
V_{p} \\
I_{p} \\
V_{q} \\
I_{q}
\end{array}\right]=\left[\begin{array}{l}
0 \\
0
\end{array}\right]
$$

where $Z_{p}$ and $Z_{q}$ are the primary and secondary winding impedances respectively, $Z_{m}$ is the magnetising impedance and $a$ is the ratio of turns between the primary and secondary winding such that:

$$
a=\frac{V_{p}}{V_{s}}=\frac{I_{s}}{I_{p}}
$$

IIDG units do not possess an inherent inertia. The dynamics of the inverter are therefore governed by the control and protection of the IIDG unit. Within the controller, the voltage is passed ahead of the current controller through a feedforward low pass filter. The feed-forward filter has a much faster response time than the current controller; hence, the IIDG unit will response to changes in voltage must faster than changes in current. From a DN protection perspective, the change in voltage as a result of a fault can be assumed to be instantaneous. The current injected by an IIDG unit will gradually change until the power reference set points allocated within the control scheme are realised. Assuming that the voltage does not change significantly as the current controller regulates the power export by the IIDG and the protection does not operate, the fault response of an IIDG can be accurately represented by (6).

$$
i(t)=\frac{S_{\mathrm{ref}}^{*}}{3 V_{L N}^{*}}+\left(I_{\mathrm{IDG}_{0}}-\frac{S_{\mathrm{ref}}^{*}}{3 V_{L N}^{*}}\right) \exp ^{-\omega_{c} t}
$$

Where $\omega_{c}$ is the cut-off frequency of the LPF and $S_{\text {ref }}$ is the power set-point of an IIDG as shown in Fig. 1. $V_{L N}$ is the line-to-neutral voltage and the IIDG's point of common coupling. $I_{\mathrm{IIDG}_{0}}$ is the current supplied by the IIDG at the fault instant.

In order to utilise (6) to approximate the fault response, the pre-fault voltage of each IIDG unit $V_{L N_{\text {prefault }}}$ must be known as well as the steady-state current supplied by each IIDG unit. In order to find the voltage at the fault instant, each IIDG unit behaves as a constant current source where the current is defined by (7).

$$
I_{\mathrm{IIDG}}=\frac{S_{\mathrm{ref}}^{*}}{3 V_{L N_{\text {pre-fault }}^{*}}}
$$

The characteristic equation of the IIDG can then be determined by (8).

$$
\left[\begin{array}{ll}
0 & 1
\end{array}\right]\left[\begin{array}{c}
V_{a} \\
I_{a}
\end{array}\right]=\left[I_{I I D G}\right]
$$

Upon concatenating the characteristic equations with $\mathrm{KCL}$ and KVL equations, the conditions at the fault instant can be determined. The algorithms used to derive the KCL and KVL equations are omitted here due to the complexity of the KVL algorithm.

The FAT presented in this paper uses an approximation of the initial current of IIDG units and a novel solver based on Newtonian methods to determine the expected fault response. The first step in determining the steady state currents of the IIDG is to find the error in the power export of each IIDG unit which is calculated by (9);

$$
\Delta S_{k}=\Delta P_{k}+j \Delta Q_{k}=-S_{\text {ref }_{k}}+\sum_{\alpha=a, b, c} V_{\alpha_{k}} I_{\alpha_{k}}^{*}
$$

where $k$ is an IIDG unit and $\alpha=a, b, c$ is used as a partial sum iterative variable across all three phases. The first iteration uses the data from the fault instant. Each successive iteration will correct the output current vector of each IIDG until the total power error $\Sigma|\Delta S|$ is acceptably small.

The $A$ and $x$ matrices constructed for (1) will be reused for determination of the partial derivatives of $x$ with respect to 
the IIDG current magnitude and phase. However, the matrix of constants will require alteration as the partial derivatives of constants are all equal to zero, with the exception of the elements $k$ which specify the IIDG currents. The altered matrix of constants is designated as $C$. When finding the partial derivative with respect to the IIDG current magnitude, the corresponding non-zero constant matrix elements can be calculated as shown in (10).

$$
C_{k}=\frac{I_{k}}{\left|I_{k}\right|}
$$

The partial derivatives of the network voltage and currents with respect to the IIDG current magnitudes can then be calculated by (11).

$$
A \frac{\partial x}{\partial\left|I_{k}\right|}=C
$$

The Jacobian elements can then be calculated by (12).

$$
\frac{\partial S_{k}}{\partial\left|I_{k}\right|}=\frac{I_{k}^{*}}{\left|I_{k}\right|} \sum_{\alpha=a, b, c} V_{k \alpha}\left(I_{k}\right)+I_{k}^{*} \sum_{\alpha=a, b, c} \frac{\partial V_{k \alpha}\left(I_{k}\right)}{\partial\left|I_{k}\right|}
$$

All non-diagonal elements are equal to zero. The same method can be used to determine the partial derivatives of the network with respect to the current phase using (13), (14) and (15).

$$
\begin{gathered}
C_{k}=j I_{k} \\
A \frac{\partial x}{\partial \delta_{k}}=C \\
\frac{\partial S_{k}}{\partial \delta_{I k}}=-j I_{k}^{*} \sum_{\alpha=a, b, c} V_{k \alpha}\left(I_{k}\right)+I_{k}^{*} \sum_{\alpha=a, b, c} \frac{\partial V_{k \alpha}\left(I_{k}\right)}{\partial \delta_{k}}
\end{gathered}
$$

The complete Jacobian matrix can then be constructed as shown in (16) and the changes to the IIDG current export can be calculated and implemented using (17).

$$
\begin{aligned}
{\left[\begin{array}{c}
\Delta\left|I_{k}\right| \\
\Delta \delta_{I_{k}}
\end{array}\right] } & =\left[\begin{array}{ll}
\mathfrak{R e}\left(\frac{\partial S_{k}}{\partial\left|I_{k}\right|}\right) & \mathfrak{R e}\left(\frac{\partial S_{k}}{\partial \delta_{I k}}\right) \\
\mathfrak{I m}\left(\frac{\partial S_{k}}{\partial\left|I_{k}\right|}\right) & \mathfrak{I m}\left(\frac{\partial S_{k}}{\partial \delta_{I k}}\right)
\end{array}\right]^{-1}\left[\begin{array}{c}
\Delta P_{k} \\
\Delta Q_{k}
\end{array}\right] \\
I_{k_{n}} & =\left(\left|I_{k_{n-1}}\right|-\Delta\left|I_{k}\right|\right) \exp ^{j\left(\delta_{k_{n-1}}-\Delta \delta_{I_{k}}\right)}
\end{aligned}
$$

When the differences between the power exports by the IIDG and the specified set points are acceptably small, the FAT can be terminated. The gathered data can then be used to extrapolate the pre-fault, transient and steady-state response of each IIDG unit using (6). Provisions should be made for an escape sequence if convergence is not achieved within a set number of iterations. The authors found that convergence is generally achieved in less than ten iterations.

\section{Simulation}

A simulation of an example network shown in Fig. 3 was implemented to verify the FAT against an equivalent smallsignal model. Fig. 3 is a single line diagram of a three-phase system.

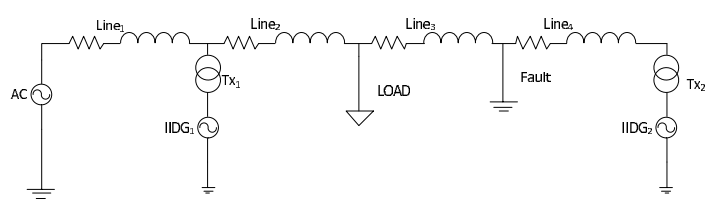

Fig. 3: Example Network

The details of the example network are given in the appendix.

\section{A. Simulations}

The first simulation involved a single-phase to earth fault on phase $A$ at $0.15 \mathrm{~s}$. The results of the simulation are given in Fig. 4, 5, 6 and 7. The sinusoidal waveforms are the results of the small signal model and the exponential responses are the voltage and current magnitude outputs of the FAT.

The second simulation involved a line-to-line fault at the same location between phases $\mathrm{A}$ and $\mathrm{B}$ at $0.15 \mathrm{~s}$. The results of the simulation are given in Fig. 8, 9, 10 and 11.

\section{B. Analysis}

The results show that the FAT data closely matches the equivalent small signal model which adopts the control scheme given in Fig. 1. As expected, the transient response is dominated by the low pass filter. The small-signal model shows a very short term fault current excursion for IIDG2 in both simulations that is not observed by the FAT. The sub-transient response is not observed by IIDG1 due to the increased electrical distance between the IIDG1 and the fault. The most notable anomaly is the fault current response of IIDG2 during simulation II. Line commutation can only be achieved through the $\mathrm{C}$ phase connected through the delta-wye transformer. The result is a heavily distorted signal; however, the FAT still provides a reasonable approximation for the

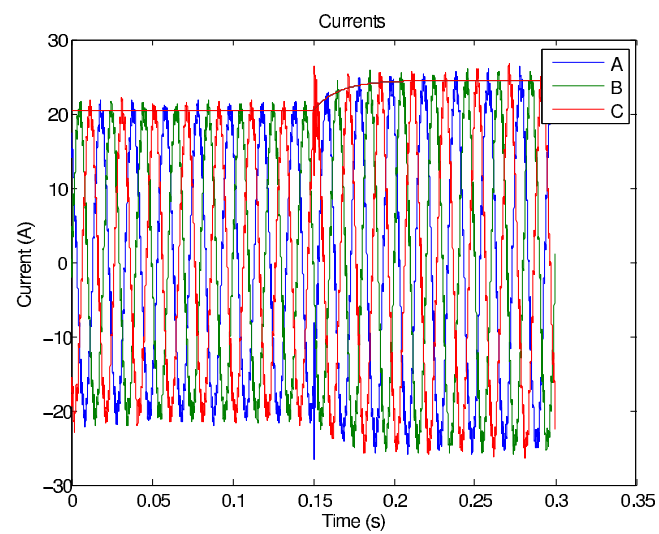

Fig. 4: Simulation I: IIDG1 Current Comparison 


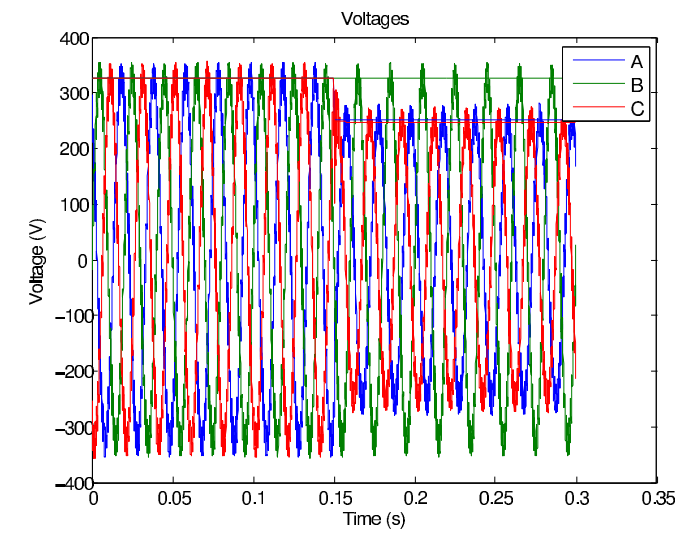

Fig. 5: Simulation I: IIDG1 Voltage Comparison

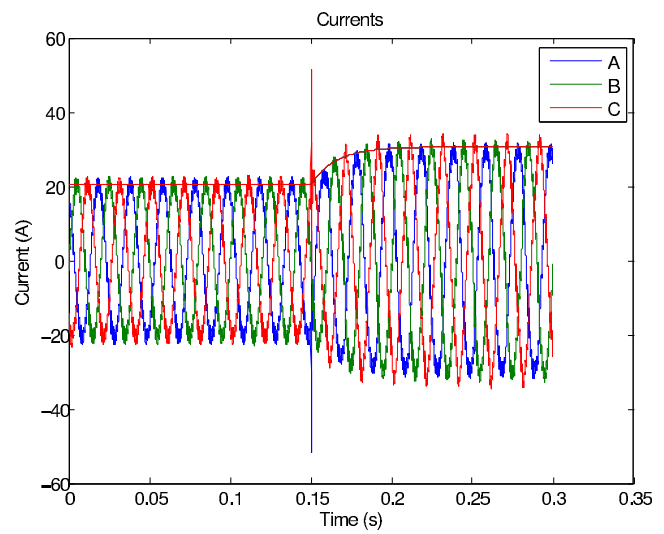

Fig. 6: Simulation I: IIDG2 Current Comparison

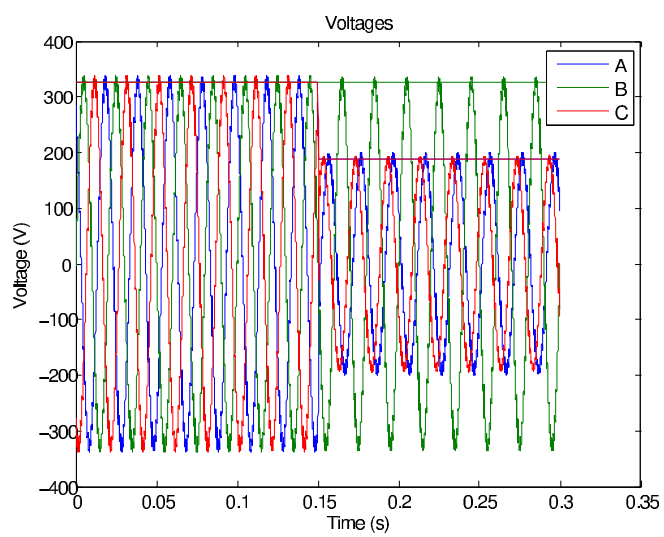

Fig. 7: Simulation I: IIDG2 Voltage Comparison

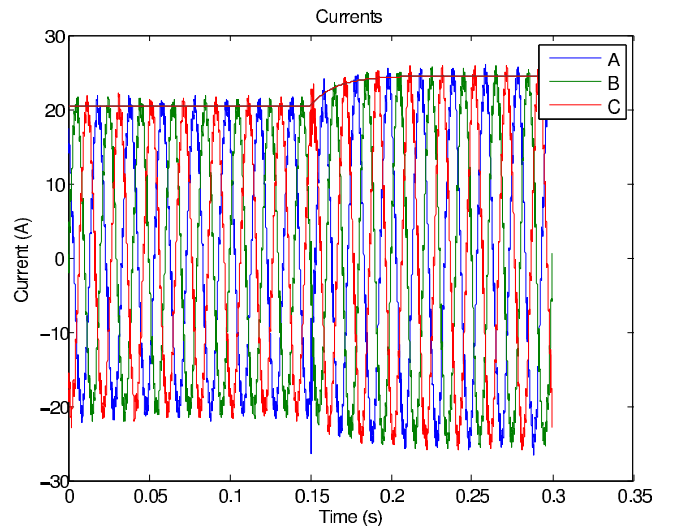

Fig. 8: Simulation II: IIDG1 Current Comparison

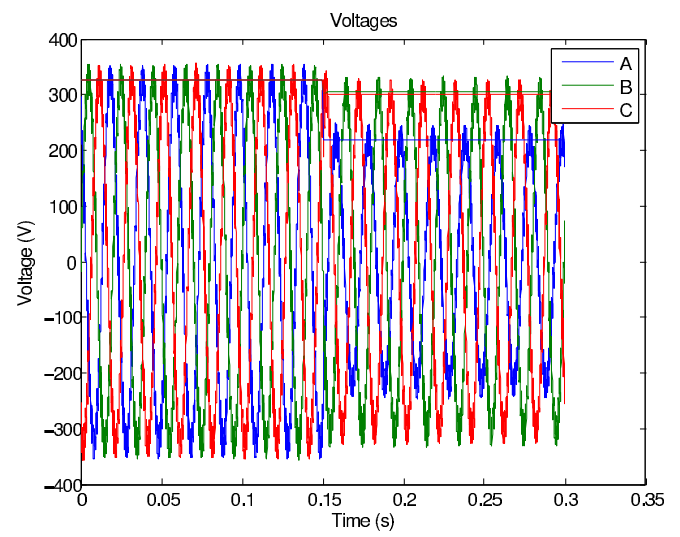

Fig. 9: Simulation II: IIDG1 Voltage Comparison

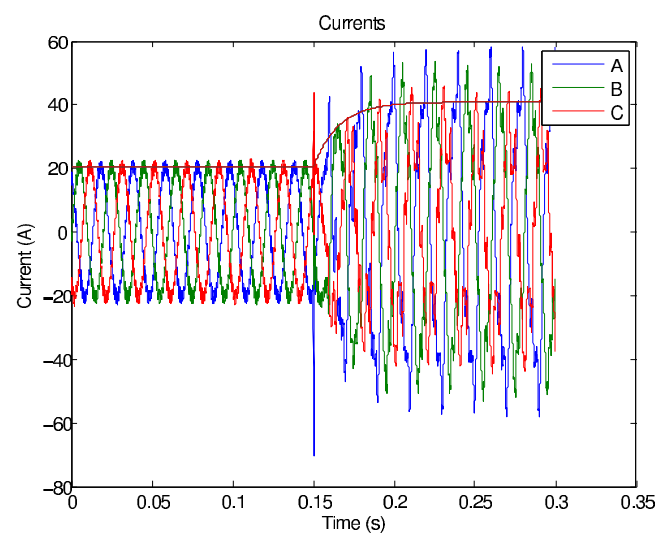

Fig. 10: Simulation II: IIDG2 Current Comparison 


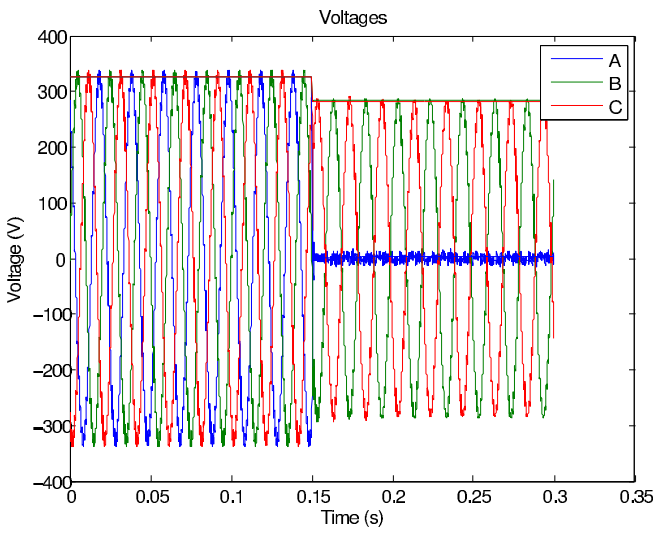

Fig. 11: Simulation II: IIDG2 Voltage Comparison

fundamental component of the resultant current waveform.

The peak current excursion of IIDG2 is approximately 2.5 times the peak rated current and has a duration of approximately 1 millisecond. The observed results are commensurate with the data gathered by [8] under laboratory conditions. The fault current observed within the first millisecond can be likened to the sub-transient response of a synchronous machine. The 'sub-transient' response of an IIDG is important for the protection of the power electronics within the inverter. Hence, the FAT is inappropriate for over-current protection of IIDG devices unless an IIDG contains a significant thermal inertia. The primary function of the FAT is to approximate the fault response for the purpose of DN line protection design.

Modern DN protection devices pass fault current data through a anti-aliasing filter, often a cosine filter, before analysing the waveform [10]. Cosine filters effectively eliminate high frequency transients; the 'sub-transient' response of an inverter will not be observed by $\mathrm{DN}$ protection devices. Hence, the omission of the 'sub-transient' response of an IIDG unit is unimportant from the perspective of DN protection analysis.

e The fault current and voltage of an IIDG unit calculated by the FAT is dictated by the response of the control system. IIDG units are incapable of sustaining over-current and over-voltage; protection systems are designed such that an IIDG unit will be isolated before thermal breakdown of the power electronics switches occurs. The FAT can be linked with protection settings such that the fault response of a network is recalculated each time a protection device operates. The fault current contribution of an IIDG can be removed by simply setting the output current and power set points to zero or by removing the IIDG from the characteristics, KVL and KCL equations.

\section{CONCLUSION}

The FAT is an effective means of approximating the fault response of IIDG in DN from the perspective of DN line protection. It must be emphasised that FAT may not be appropriate for analysing the efficacy of IIDG protection, in particular the effects of the 'sub-transient' response and islanded modes of operation on local protection. Future work will involve incorporating the protection settings of DN protection devices into the FAT. Also, an investigation into the possibility of modelling the sub-transient response of the IIDG without requiring a significant knowledge of the control dynamics of the IIDG would be of significant value. Finally, extending the FAT into intentional islanding may prove useful for microgrid applications.

\section{APPENDIX}

\begin{tabular}{|c|c|c|c|c|}
\hline \multicolumn{5}{|c|}{ Infinite Bus Data } \\
\hline Voltage 1-1 rms (kV) & \multicolumn{4}{|c|}{11000} \\
\hline Frequency $(\mathrm{Hz})$ & \multicolumn{4}{|c|}{50} \\
\hline \multicolumn{5}{|c|}{ Line Data } \\
\hline Line No. & 1 & 2 & 3 & 4 \\
\hline Resistance $(\Omega)$ & 0.3 & 0.3 & 0.3 & 0.003 \\
\hline Inductance $(\mathrm{mH})$ & 3 & 3 & 3 & 0.3 \\
\hline \multicolumn{5}{|c|}{ Transformer Data } \\
\hline Transformer No. & & 1 & & 2 \\
\hline Primary Voltage 1-1 rms (V) & & 11000 & & 11000 \\
\hline Primary Winding Resistance $(\Omega)$ & & 0.003 & & 0.0003 \\
\hline Primary Winding Inductance $(\mathrm{mH})$ & & 1 & & 0.5 \\
\hline Primary Connection & & Delta & & Delta \\
\hline Secondary Voltage 1-1 rms (V) & & 400 & & 400 \\
\hline Secondary Winding Resistance $(\Omega)$ & & 0.003 & & 0.0001 \\
\hline Secondary Winding Inductance $(\mathrm{mH})$ & & 1 & & 0.2 \\
\hline Secondary Connection & & ye-earthed & & ye-earthed \\
\hline \multicolumn{5}{|c|}{ Load Data } \\
\hline Resistance $(\Omega)$ & \multicolumn{4}{|c|}{605} \\
\hline Inductance $(\mathrm{H})$ & \multicolumn{4}{|c|}{38.5155} \\
\hline \multirow{2}{*}{\multicolumn{5}{|c|}{$\begin{array}{c}\text { Note: The equivalent resistance and inductance of the load are connected in parallel. } \\
\text { IIDG Data }\end{array}$}} \\
\hline & & & & \\
\hline \multicolumn{5}{|c|}{\begin{tabular}{|c|c|}
\multicolumn{4}{|c|}{ IIDG Data } \\
IIDG No.
\end{tabular}} \\
\hline DC Voltage (V) & & 800 & & 800 \\
\hline Switching Frequency $(\mathrm{kHz})$ & & 4 & & 4 \\
\hline Filter Inductance $(\mathrm{mH})$ & & 16.33 & & 16.33 \\
\hline Filter Capacitance $(\mu \mathrm{F})$ & & 3 & & 3 \\
\hline Power Setpoint (kVA) & & $10+\mathrm{j} 0$ & & $10+\mathrm{j} 0$ \\
\hline Low Pass Filter Cut-Off Frequency (Hz) & & 10 & & 10 \\
\hline \multicolumn{5}{|c|}{ Fault Data } \\
\hline Simulation No. & & I & & II \\
\hline Fault time $(\mathrm{s})$ & & 0.15 & & 0.15 \\
\hline Fault type & $\overline{\mathrm{A} \mathrm{p}}$ & ase to earth & $\overline{\mathrm{A} \mathrm{pl}}$ & ase to $B$ phase \\
\hline Fault impedance $(\Omega)$ & & $0001+\mathrm{j} 0$ & & $0.001+\mathrm{j} 0$ \\
\hline
\end{tabular}

\section{REFERENCES}

[1] Wijnhoven, T.; Tant, J.; Deconinck, G., "Inverter modelling techniques for protection studies", Power Electronics for Distributed Generation Systems (PEDG), 2012 3rd IEEE International Symposium on , vol., no., pp.187,194, 25-28 June 2012

[2] Plet, C.A; Graovac, M.; Green, T.C.; Iravani, R., "Fault response of grid-connected inverter dominated networks," Power and Energy Society General Meeting, 2010 IEEE, vol., no., pp.1,8, 25-29 July 2010

[3] Soultanis, N.L.; Papathanasiou, S.A; Hatziargyriou, N.D., "A Stability Algorithm for the Dynamic Analysis of Inverter Dominated Unbalanced LV Microgrids," Power Systems, IEEE Transactions on , vol.22, no.1, pp.294,304, Feb. 2007

[4] A. Yazdani and R. Iravani, "Voltage-Sourced Converters in Power Systems", New York: Wiley, 2010

[5] Alaboudy, AH.K.; Zeineldin, H.H., "Islanding Detection for InverterBased DG Coupled With Frequency-Dependent Static Loads," Power Delivery, IEEE Transactions on , vol.26, no.2, pp.1053,1063, April 2011

[6] Van Tu, D., Chaitusaney, S. and Yokoyama, A. (2013), "Fault current calculation in distribution systems with inverter-based distributed generations," IEEJ Trans Elec Electron Eng, 8: 470477 
[7] Loix, T.; Wijnhoven, T.; Deconinck, G., "Protection of microgrids with a high penetration of inverter-coupled energy sources", Integration of Wide-Scale Renewable Resources Into the Power Delivery System, 2009 CIGRE/IEEE PES Joint Symposium , vol., no., pp.1,6, 29-31 July 2009

[8] J. Keller and B. Kroposki, "Understanding Fault Characteristics of Inverter-Based Distributed Energy Resources", National Renewable Energy Laboratory, NREL/TP-550-46698, Jan 2010

[9] Dash, AR.; Babu, B.C.; Mohanty, K.B.; Dubey, R., "Analysis of PI and PR controllers for distributed power generation system under unbalanced grid faults," Power and Energy Systems (ICPS), 2011 International Conference on , vol., no., pp.1,6, 22-24 Dec. 2011

[10] E. O. Schweitzer III and Daqing Hou, "Filtering for Protective Relay," 19th Annual Western Protective Relay Conference, Spokane, Washington, October 20-22, 1992. 